Теорія Ймовір. та Матем. Статист. Вип. 79, 2008
Theor. Probability and Math. Statist.

No. 79, 2009, Pages 83-88

S 0094-9000(09)00782-0

Article electronically published on December 29, 2009

\title{
APPROXIMATION OF $\operatorname{SSub}_{\varphi}(\Omega)$ STOCHASTIC PROCESSES \\ IN THE SPACE $L_{p}(\mathbb{T})$
}

UDC 519.21

\author{
YU. V. KOZACHENKO AND O. E. KAMENSHCHIKOVA
}

\begin{abstract}
A bound for the distributions of norms is obtained for $\operatorname{Sub}_{\varphi}(\Omega)$ stochastic processes in the space $L_{p}(\mathbb{T})$. This bound is used to construct an approximation of strictly $\varphi$-sub-Gaussian processes by random broken lines in the space $L_{p}(\mathbb{T})$ with a given accuracy and reliability
\end{abstract}

\section{INTRODUCTION}

We consider an approximation of strictly $\varphi$-sub-Gaussian stochastic processes by random broken lines in the space $L_{p}(\mathbb{T})$. We obtain an upper bound for the norm of $\operatorname{Sub}_{\varphi}(\Omega)$ processes and use it to construct an approximation of a stochastic process with a given accuracy and reliability.

We recall some definitions and results concerning $\operatorname{Sub}_{\varphi}(\Omega)$ and $\operatorname{SSub}_{\varphi}(\Omega)$ stochastic processes.

The notion of the $\operatorname{Sub}_{\varphi}(\Omega)$ space is introduced in the paper [3. The definition and properties of $\operatorname{Sub}_{\varphi}(\Omega)$ random variables are studied in [2].

Let $(\Omega, B, \mathrm{P})$ be a standard probability space.

Definition 1.1 (1]). A continuous even convex function $u=(u(x), x \in \mathbf{R})$ is called an $N$-Orlicz function if it increases in the domain $x>0, u(0)=0$, and if

$$
\frac{u(x)}{x} \rightarrow 0 \quad \text { as } x \rightarrow 0 \quad \text { and } \quad \frac{u(x)}{x} \rightarrow \infty \quad \text { as } x \rightarrow \infty .
$$

Condition $\mathbf{Q}([2])$. We say that a function $N$ satisfies condition $\mathbf{Q}$ if

$$
\varliminf_{x \rightarrow 0} \frac{\varphi(x)}{x^{2}}=c>0 .
$$

Remark 1.1. The case of $c=\infty$ in Condition $\mathbf{Q}$ also fits Definition 1.1.

Definition $1.2([2])$. Let $\varphi$ be an $N$-Orlicz function satisfying condition Q. We say that a centered random variable $\xi$ belongs to $\operatorname{Sub}_{\varphi}(\Omega)$ (to the space of $\varphi$-sub-Gaussian random variables) if there exists a constant $r_{\xi} \geq 0$ such that

$$
\operatorname{E} \exp (\lambda \xi) \leq \exp \left(\varphi\left(r_{\xi} \lambda\right)\right)
$$

for all $\lambda \in \mathbf{R}$.

Theorem $1.1([1]-[3]) \cdot \operatorname{Sub}_{\varphi}(\Omega)$ is a Banach space with respect to the norm

$$
\tau_{\varphi}(\xi)=\inf \{a \geq 0: \operatorname{Exp}(\lambda \xi) \leq \exp (\varphi(a \lambda)), \lambda \in \mathbf{R}\} .
$$

2000 Mathematics Subject Classification. Primary 60G17; Secondary 60 G07.

Key words and phrases. Approximation, $\operatorname{SSub}_{\varphi}(\Omega)$ process, interpolating broken line. 
YU. V. KOZACHENKO AND O. E. KAMENSHCHIKOVA

Definition $1.3([1])$. Let $\mathbb{T}$ be a set of parameters. We say that a stochastic pro-

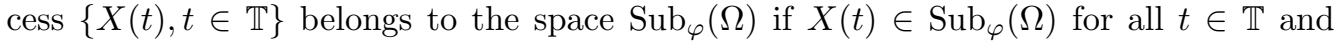
$\sup _{t \in \mathbb{T}} \tau_{\varphi}(X(t))<\infty$.

Definition $1.4\left([4)\right.$. A family $\Delta$ of random variables $\xi$ of the $\operatorname{space} \operatorname{Sub}_{\varphi}(\Omega)$ is called a strictly $\operatorname{Sub}_{\varphi}(\Omega)$ family if there exists a constant $C_{\Delta}>0$ such that

$$
\tau_{\varphi}\left(\sum_{i \in I} \lambda_{i} \xi_{i}\right) \leq C_{\Delta}\left(\mathrm{E}\left(\sum_{i \in I} \lambda_{i} \xi_{i}\right)^{2}\right)^{1 / 2}
$$

for all finite sets $I$, for all collections $\xi_{i} \in \Delta, i \in I$, and for all $\lambda_{i} \in \mathbf{R}^{1}$.

The number $C_{\Delta}$ is called a defining constant.

Definition $1.5([4)$. A $\varphi$-sub-Gaussian stochastic process $\{X(t), t \in \mathbb{T}\}$ is called a strictly $\operatorname{Sub}_{\varphi}(\Omega)$ process if the family of random variables $\{X(t), t \in \mathbb{T}\}$ is a strictly $\operatorname{Sub}_{\varphi}(\Omega)$ family.

Definition 1.6 (1]). Let $f=(f(x), x \in \mathbf{R})$ be a real function. The function

$$
f^{*}=\left(f^{*}(x), x \in \mathbf{R}\right)
$$

defined by $f^{*}(x)=\sup _{y \in \mathbf{R}}(x y-f(y))$ is called the Young-Fenchel transform of the function $f$ or the conjugate function to $f$.

Let $X=\{X(t), t \in \mathbb{T}\}, \mathbb{T}=[0, T]$, be a given strictly $\varphi$-sub-Gaussian stochastic process with a defining constant $C_{\Delta}$.

Let $S:=\left\{t_{k}\right\}_{k=0}^{k=N}=\{T k / N, k=0, \ldots, N\}$ be a partition of the interval $\mathbb{T}$ into $N$ parts $\left[t_{k}, t_{k+1}\right]$. We want to approximate the process $\{X(t), t \in \mathbb{T}\}$ by the line $X_{N}(t)$ constructed from the points $\left(t_{k}, X\left(t_{k}\right)\right), k=0, \ldots, N$, as follows:

$$
X_{N}(t)=\alpha_{1} X\left(t_{k}\right)+\alpha_{2} X\left(t_{k+1}\right), \quad t \in\left[t_{k}, t_{k+1}\right], k=0, \ldots, N-1,
$$

where $\alpha_{1}=1-\left(t-t_{k}\right) N / T$ and $\alpha_{2}=\left(t-t_{k}\right) N / T$.

Definition 1.7. We say that a broken line $X_{N}(t)$ approximates the process $X(t)$ in the space $L_{p}(\mathbb{T})$ with a given accuracy $\varepsilon>0$ and reliability $1-\delta, 0<\delta<1$, if

$$
\mathrm{P}\left\{\left(\int_{\mathbb{T}}\left|X(t)-X_{N}(t)\right|^{p} d t\right)^{1 / p}>\varepsilon\right\} \leq \delta .
$$

The problem considered in this paper is to reconstruct the process $\{X(t)\}$ from the broken line $\left\{X_{N}(t), t \in \mathbb{T}\right\}$ with a given accuracy $\varepsilon>0$ and reliability $1-\delta, 0<\delta<1$, in the norm of the space $L_{p}(\mathbb{T})$ if the values of the process at the corresponding points $\{T k / N, k=0, \ldots, N\}$ are known.

Let $Y_{N}(t):=X(t)-X_{N}(t), t \in \mathbb{T}$, be the deviation process.

Let the process $\{X(t), t \in \mathbb{T}\}$ be such that

$$
\mathrm{E}|X(t+h)-X(t)|^{2} \leq b^{2}(h) \text { for all } h>0 \text { and for all } t \in \mathbb{T} \text {, }
$$

where $b(h)$ increases and $b(h) \rightarrow 0$ as $h \rightarrow 0$.

2. A BOUND FOR NORMS IN $L_{p}(\mathbb{T})$ FOR SUb-Gaussian STOChastiC PROCESSES

Theorem 2.1. Let $\{\mathbb{T}, \Lambda, M\}$ be a measurable space, $X=\{X(t), t \in \mathbb{T}\}$ a measurable stochastic process belonging to the space $\operatorname{Sub}_{\varphi}(\Omega)$, and let $\tau_{\varphi}(t):=\tau_{\varphi}(X(t))$. Further, let the Lebesgue integral $\int_{\mathbb{T}}\left(\tau_{\varphi}(t)\right)^{p} d \mu(t)<\infty$ be well defined for $p \geq 1$. Then the integral $\int_{\mathbb{T}}|X(t)|^{p} d \mu(t)<\infty$ exists with probability 1 and

$$
\mathrm{P}\left\{\int_{\mathbb{T}}|X(t)|^{p} d \mu(t)>\varepsilon\right\} \leq 2 \exp \left\{-\varphi^{*}\left(\left(\frac{\varepsilon}{c}\right)^{1 / p}\right)\right\}
$$


for all $\varepsilon>c\left(f\left(c^{1 / p} p / \varepsilon^{1 / p}\right)\right)^{p}$, where $c=\int_{\mathbb{T}}\left(\tau_{\varphi}(t)\right)^{p} d \mu(t), f$ is a function such that $\varphi(u)=\int_{0}^{u} f(v) d v$ for $u>0$, and where $\varphi^{*}$ is the Young-Fenchel transform of the function $\varphi$.

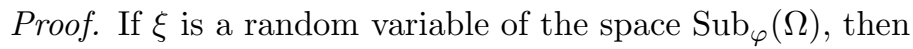

$$
\mathrm{E}|\xi|^{\alpha} \leq 2\left(\frac{\alpha}{e}\right)^{\alpha}\left(\tau_{\varphi}(\xi)\right)^{\alpha} \cdot \exp \{\varphi(\lambda)-\alpha \ln \lambda\}
$$

for arbitrary $\alpha>0$ and $\lambda>0$ (see [2]). Inequality (2) together with Fubini's theorem implies that

$$
\begin{aligned}
\mathrm{E} \int_{\mathbb{T}}|X(t)|^{p} d \mu(t) & =\int_{\mathbb{T}} \mathrm{E}|X(t)|^{p} d \mu(t) \leq 2\left(\frac{p}{e}\right)^{p} \int_{\mathbb{T}}\left(\tau_{\varphi}(t)\right)^{p} d \mu(t) \exp \{\varphi(\lambda)-p \ln \lambda\} \\
& <\infty
\end{aligned}
$$

for all $p \geq 1$ and $\lambda>0$; that is, $\int_{\mathbb{T}}|X(t)|^{p} d \mu(t)<\infty$ with probability 1 .

Let $r \geq 1$. Then the generalized Minkowski inequality implies that

$$
\begin{aligned}
\left\|\int_{\mathbb{T}}|X(t)|^{p} d \mu(t)\right\|_{L_{r}} & =\left(\left.\left.\mathrm{E}\left|\int_{\mathbb{T}}\right| X(t)\right|^{p} d \mu(t)\right|^{r}\right)^{1 / r} \leq \int_{\mathbb{T}}\left\||X(t)|^{p}\right\|_{L_{r}} d \mu(t) \\
& =\int_{\mathbb{T}}\left(\mathrm{E}|X(t)|^{p r}\right)^{1 / r} d \mu(t) .
\end{aligned}
$$

Now we obtain from inequality (2) that

$$
\begin{aligned}
\left\|\int_{\mathbb{T}}|X(t)|^{p} d \mu(t)\right\|_{L_{r}} & \leq \int_{\mathbb{T}}\left(2\left(\tau_{\varphi}(t)\right)^{p r}\left(\frac{p r}{e}\right)^{p r} \exp \{\varphi(\lambda)\} \lambda^{-p r}\right)^{1 / r} d \mu(t) \\
& =\left(2\left(\frac{p r}{e}\right)^{p r} \exp \{\varphi(\lambda)\} \lambda^{-p r}\right)^{1 / r} \int_{\mathbb{T}}\left(\tau_{\varphi}(t)\right)^{p} d \mu(t)
\end{aligned}
$$

for all $\lambda>0$. Let $\varepsilon>0, r \geq 1$, and $\lambda>0$. Using the Chebyshev inequality we derive from (3) that

$$
\begin{aligned}
\mathrm{P}\left\{\int_{\mathbb{T}}|X(t)|^{p} d \mu(t)>\varepsilon\right\} & \leq \frac{\left(\mathrm{E} \int_{\mathbb{T}}|X(t)|^{p} d \mu(t)\right)^{r}}{\varepsilon^{r}} \\
& \leq 2\left(\frac{p r}{e}\right)^{p r} \frac{\lambda^{-p r} \exp \{\varphi(\lambda)\}}{\varepsilon^{r}}\left(\int_{\mathbb{T}}\left(\tau_{\varphi}(t)\right)^{p} d \mu(t)\right)^{r} .
\end{aligned}
$$

Letting $c:=\int_{\mathbb{T}}\left(\tau_{\varphi}(t)\right)^{p} d \mu(t)$, we deduce from inequality (4) that

$$
\mathrm{P}\left\{\int_{\mathbb{T}}|X(t)|^{p} d \mu(t)>\varepsilon\right\} \leq 2\left(\frac{c}{\varepsilon} \frac{p^{p}}{(\lambda e)^{p}}\right)^{r} \cdot r^{p r} \exp \{\varphi(\lambda)\}=2 a^{r} r^{p r} \exp \{\varphi(\lambda)\},
$$

where $a=c p^{p} /\left(\varepsilon(\lambda e)^{p}\right)$.

Let $\psi(r)=a^{r} r^{p r}$. Then

$$
(\ln \psi(r))^{\prime}=(r \ln a+p r \ln r)^{\prime}=\ln a+p \ln r+p .
$$

Thus $(\ln \psi(r))^{\prime}=0$ if $r=a^{-1 / p} e^{-1}$. For this point $r$,

$$
\psi\left(a^{-1 / p} e^{-1}\right)=\exp \left\{-p a^{-1 / p} e^{-1}\right\},
$$

whence

$$
\begin{aligned}
\mathrm{P}\left\{\int_{\mathbb{T}}|X(t)|^{p} d \mu(t)>\varepsilon\right\} & \leq 2 \exp \left\{-\left(\frac{p a^{-1 / p}}{e}-\varphi(\lambda)\right)\right\} \\
& =2 \exp \left\{-\left(\lambda\left(\frac{\varepsilon}{c}\right)^{1 / p}-\varphi(\lambda)\right)\right\}
\end{aligned}
$$


if $a^{-1 / p} e^{-1}>1$, that is, if $\varepsilon^{1 / p} \lambda /\left(c^{1 / p} p\right) \geq 1$ or $\lambda \geq c^{1 / p} p / \varepsilon^{1 / p}=z$. Inequality (5) implies that

$$
\mathrm{P}\left\{\int_{\mathbb{T}}|X(t)|^{p} d \mu(t)>\varepsilon\right\} \leq 2 \exp \left\{-\sup _{\lambda \geq z}\left(\lambda\left(\frac{\varepsilon}{c}\right)^{1 / p}-\varphi(\lambda)\right)\right\} .
$$

Let $\varphi^{*}(u)$ be the Young-Fenchel transform of the function $\varphi(u)$ for $u>0$. This means that

$$
\varphi^{*}(u)=\sup _{\lambda>0}(u \lambda-\varphi(\lambda)) .
$$

The supremum in equality (7) is attained at the point

$$
\lambda=f^{(-1)}(u)
$$

(see [1]), where $f^{(-1)}(u)$ is the generalized inverse function to $f(u)$ and $f(u)$ is the density of $\varphi(u)$, that is, $\varphi(u)=\int_{0}^{u} f(v) d v$.

Thus inequality (6) implies that

$$
\mathrm{P}\left\{\int_{\mathbb{T}}|X(t)|^{p} d \mu(t)>\varepsilon\right\} \leq 2 \exp \left\{-\varphi^{*}\left(\left(\frac{\varepsilon}{c}\right)^{1 / p}\right)\right\}
$$

for

$$
f^{(-1)}\left(\left(\frac{\varepsilon}{c}\right)^{1 / p}\right) \geq z
$$

Note that inequality (8) holds if

$$
\left(\frac{\varepsilon}{c}\right)^{1 / p} \geq f\left(\frac{c^{1 / p} p}{\varepsilon^{1 / p}}\right)
$$

that is, if

$$
\varepsilon \geq c\left(f\left(\frac{c^{1 / p} p}{\varepsilon^{1 / p}}\right)\right)^{p}
$$

Corollary 2.1. If $\varphi(x)=x^{2} / 2$, that is, if $X(t)$ is a sub-Gaussian stochastic process, then inequality (11) becomes of the following form:

$$
\mathrm{P}\left\{\int_{\mathbb{T}}|X(t)|^{p} d \mu(t)>\varepsilon\right\} \leq 2 \exp \left\{-\frac{\varepsilon^{2 / p}}{2 c^{2 / p}}\right\}
$$

for $\varepsilon>c p^{p / 2}$, where

$$
c=\int_{\mathbb{T}}\left(\tau_{x^{2} / 2}(t)\right)^{p} d \mu(t) .
$$

\section{ACCURACY AND RELIABILITy of the APproximation}

Lemma 3.1. Let $X(t)$ be a $\operatorname{SSub}_{\varphi}(\Omega)$ process with a defining constant $C_{\Delta}$,

$$
\mathrm{E}|X(t+h)-X(t)|^{2} \leq b^{2}(h) \quad \text { for all } h>0 \text { and for all } t \in \mathbb{T},
$$

where $b(h)$ increases and $b(h) \rightarrow 0$ as $h \rightarrow 0$. Then the deviation process $\left\{Y_{N}(t), t \in \mathbb{T}\right\}$ is such that

$$
\tau_{\varphi}\left(Y_{N}(t)\right) \leq C_{\Delta} b\left(\frac{T}{N}\right) \quad \text { for all } t \in \mathbb{T}
$$


Proof. We apply the inequality $\tau_{\varphi}\left(Y_{N}(t)\right) \leq C_{\Delta}\left(\mathrm{E} Y_{N}^{2}(t)\right)^{1 / 2}$. Taking into account the representation

$$
X_{N}(t)=\alpha_{1} X\left(t_{k}\right)+\alpha_{2} X\left(t_{k+1}\right), \quad t \in\left[t_{k}, t_{k+1}\right], \quad k=0, \ldots, N-1,
$$

where $\alpha_{1}=1-\left(t-t_{k}\right) N / T$ and $\alpha_{2}=\left(t-t_{k}\right) N / T$, we obtain

$$
\begin{aligned}
& \mathrm{E} Y_{N}^{2}(t)=\mathrm{E}\left|X(t)-X_{N}(t)\right|^{2}=\mathrm{E}\left|X(t)-\alpha_{1} X\left(t_{k}\right)-\alpha_{2} X\left(t_{k+1}\right)\right|^{2} \\
& =\mathrm{E}|X(t)|^{2}+\mathrm{E} \alpha_{1}^{2} X^{2}\left(t_{k}\right)+\mathrm{E} \alpha_{2}^{2} X^{2}\left(t_{k+1}\right)-2 \alpha_{1} \mathrm{E} X(t) X\left(t_{k}\right) \\
& -2 \alpha_{2} \operatorname{E} X(t) X\left(t_{k+1}\right)+2 \alpha_{1} \alpha_{2} \operatorname{E} X\left(t_{k}\right) X\left(t_{k+1}\right) \\
& =B(t, t)+\left(1-2 \frac{N}{T}\left(t-t_{k}\right)+\frac{N^{2}}{T^{2}}\left(t-t_{k}\right)^{2}\right) B\left(t_{k}, t_{k}\right) \\
& +\left(t-t_{k}\right)^{2} \frac{N^{2}}{T^{2}} B\left(t_{k+1}, t_{k+1}\right)-2 B\left(t, t_{k}\right)\left(1-\left(t-t_{k}\right) \frac{N}{T}\right) \\
& -2 B\left(t, t_{k+1}\right)\left(t-t_{k}\right) \frac{N}{T}+2\left(1-\left(t-t_{k}\right) \frac{N}{T}\right)\left(t-t_{k}\right) \frac{N}{T} B\left(t_{k}, t_{k+1}\right) \\
& =\left(1-\left(t-t_{k}\right) \frac{N}{T}\right)\left(B(t, t)-2 B\left(t, t_{k}\right)+B\left(t_{k}, t_{k}\right)\right) \\
& +\left(t-t_{k}\right) \frac{N}{T}\left(B(t, t)-2 B\left(t, t_{k+1}\right)+B\left(t_{k+1}, t_{k+1}\right)\right) \\
& +\left(\frac{N^{2}}{T^{2}}\left(t-t_{k}\right)^{2}-\frac{N}{T}\left(t-t_{k}\right)\right) B\left(t_{k}, t_{k}\right) \\
& +\left(\frac{N^{2}}{T^{2}}\left(t-t_{k}\right)^{2}-\frac{N}{T}\left(t-t_{k}\right)\right) B\left(t_{k+1}, t_{k+1}\right) \\
& +2\left(1-\left(t-t_{k}\right) \frac{N}{T}\right) \frac{N}{T}\left(t-t_{k}\right) B\left(t_{k}, t_{k+1}\right) \\
& =\left(1-\left(t-t_{k}\right) \frac{N}{T}\right) \mathrm{E}\left|X(t)-X\left(t_{k}\right)\right|^{2}+\frac{N}{T}\left(t-t_{k}\right) \mathrm{E}\left|X(t)-X\left(t_{k+1}\right)\right|^{2} \\
& +\frac{N}{T}\left(t-t_{k}\right)\left(1-\left(t-t_{k}\right) \frac{N}{T}\right)\left(-B\left(t_{k}, t_{k}\right)-B\left(t_{k+1}, t_{k+1}\right)+2 B\left(t_{k}, t_{k+1}\right)\right) \\
& \leq\left(1-\left(t-t_{k}\right) \frac{N}{T}\right) b^{2}\left(t-t_{k}\right)+\frac{N}{T}\left(t-t_{k}\right) b^{2}\left(t_{k+1}-t\right) \\
& -\frac{N}{T}\left(t-t_{k}\right)\left(1-\left(t-t_{k}\right) \frac{N}{T}\right) \mathrm{E}\left|X\left(t_{k}\right)-X\left(t_{k+1}\right)\right|^{2} \\
& \leq\left(1-\left(t-t_{k}\right) \frac{N}{T}\right) b^{2}\left(\frac{T}{N}\right)+\left(t-t_{k}\right) \frac{N}{T} b^{2}\left(\frac{T}{N}\right)-0=b^{2}\left(\frac{T}{N}\right),
\end{aligned}
$$

whence inequality (9) follows.

Theorem 3.1. Let $X=\{X(t), t \in \mathbb{T}\}$ be the stochastic process introduced in Lemma 3.1. Let a random broken line $X_{N}(t)$ approximate the process $X(t)$ with a given accuracy $\varepsilon$ and reliability $1-\delta$. Then the number $N$ satisfies the following system of inequalities:

$$
\left\{\begin{array}{l}
2 \exp \left\{-\varphi^{*}\left(\frac{\varepsilon^{1 / p}}{(\mu(\mathbb{T}))^{1 / p} C_{\Delta} b(T / N)}\right)\right\} \leq \delta, \\
\varepsilon>(\mu(\mathbb{T}))^{1 / p} C_{\Delta} b\left(\frac{T}{N}\right) f\left(\frac{(\mu(\mathbb{T}))^{1 / p} C_{\Delta} b(T / N) p}{\varepsilon}\right) .
\end{array}\right.
$$

Proof. This result is an easy corollary of Theorem 2.1 and Lemma 3.1 . 
YU. V. KOZACHENKO AND O. E. KAMENSHCHIKOVA

Example. Let $\varphi(x)=x^{\alpha} / \alpha,|x|>1, T=[0,1]$, and $C_{\Delta}=1$. Then $\varphi^{*}(x)=x^{\beta} / \beta$ for $|x|>1$, where $\alpha^{-1}+\beta^{-1}=1$.

Let $b(t, h)=b(h)=h$. Then Theorem 3.1 implies the following inequalities determining $N$ :

$$
\left\{\begin{array}{l}
2 \exp \left\{-\frac{\varepsilon^{\beta} N^{\beta}}{\beta}\right\} \leq \delta, \\
N>\frac{p^{(\alpha-1) / \alpha}}{\varepsilon} .
\end{array}\right.
$$

Let, for example, $p=3, \alpha=3, \varepsilon=0.01$, and $\delta=0.01$. Then the number of points in a partition of the interval $\mathbb{T}=[0,1]$ used for constructing an approximation of a given process $X(t)$ with a given accuracy $\varepsilon$ and reliability $1-\delta$ is such that $N \geq 7948$.

\section{BIBLIOGRAPHY}

1. V. V. Buldygin and Yu. V. Kozachenko, Metric characterization of random variables and random processes, TViMS, Kyiv, 1998; English transl., American Mathematical Society, Providence, RI, 2000. MR 1743716 (2001g:60089)

2. R. Guiliano Antonini, Yu. Kozachenko, and T. Nikitina, Spaces of $\varphi$-sub-Gaussian random variables, Rend. Accad. Naz. Sci. XL Mem. Mat. Appl. XXVII (2003), no. 5, 95-124. MR2056414 (2005f:60036)

3. Yu. Kozachenko and E. I. Ostrovskiŭ, Banach spaces of random variables of sub-Gaussian type, Teor. Veroyatnost. i Mat. Statist. 32 (1985), 42-53; English transl. in Theory Probab. Math. Statist. 32 (1986), 45-56. MR882158 (88e:60009)

4. Yu. Kozachenko and Yu. A. Koval'chuk, Boundary value problems with random initial conditions, and function series of $\operatorname{Sub}_{\varphi}(\Omega)$. I, Ukrain. Mat. Zh. 50 (1998), no. 4, 504-515; English transl. in Ukrainian Math. J. 50 (1998), no. 4, 572-585. MR1698149 (2000f:60029)

Department of Probability Theory and Mathematical Statistics, Faculty for Mechanics and Mathematics, National Taras Shevchenko University, Academician Glushkov Avenue 6 , KYIV 03127, UKRAINE

E-mail address: yvk@univ.kiev.ua

Department of Probability Theory and Mathematical Statistics, Faculty for Mechanics and Mathematics, National Taras Shevchenko University, Academician Glushkov Avenue 6 , KYIV 03127, UKRAINE

E-mail address: kamalev@gmail.com

Received 5/NOV/2007

Translated by N. SEMENOV 